\title{
Surface Micromachined Force Gauges: Uncertainty and Reliability
}

Jonathan W. Wittwer

Troy Gomm

Larry L. Howell

Ihowell@byu.edu

Follow this and additional works at: https://scholarsarchive.byu.edu/facpub

Part of the Mechanical Engineering Commons

\section{Original Publication Citation}

Wittwer, J.W., Gomm, T., and Howell, L.L., â€œSurface Micromachined Force Gauges:

Uncertainty and Reliability,â€ Journal of Micromechanics and Microengineering, Institute of Physics, Vol. 12, No. 1, pp. 13-2, 22.

\section{BYU ScholarsArchive Citation}

Wittwer, Jonathan W.; Gomm, Troy; and Howell, Larry L., "Surface Micromachined Force Gauges: Uncertainty and Reliability" (2002). Faculty Publications. 548.

https://scholarsarchive.byu.edu/facpub/548 


\title{
Surface Micromachined Force Gauges: Uncertainty and Reliability
}

\author{
Jonathan W. Wittwer, Troy Gomm, and Larry L. Howell ${ }^{1}$ \\ Department of Mechanical Engineering \\ Brigham Young University, \\ Provo, Utah 84602 \\ USA
}

\begin{abstract}
Surface micromachining of micro-electro-mechanical systems (MEMS), like all other fabrication processes, has inherent variation that leads to uncertain material and dimensional parameters. By considering the effects of these variations during the design of micro force gauges, the gauge uncertainty and reliability can be estimated. Without means of calibrating micro gauges, these effects are often significant when compared to experimental repeatability.

The general force gauge model described in this paper can be used to measure a wide range of forces, and simple design changes can lead to improved accuracy in measurement. A method of probabilistic design is described that is not limited to small beam deflections.
\end{abstract}

Keywords: micro force measurement, error analysis, uncertainty, reliability.

1. Corresponding Author. Phone: 801-378-8037; Fax: 801-378-5037;

E-mail: lhowell@et.byu.edu 


\section{INTRODUCTION}

Many micro mechanisms require force measurements to determine the behavior of a system or validate theoretical predictions. Friction forces, actuator forces, and contact forces are some examples of the important forces to be measured in MEMS. Fabrication of tools for measuring micro forces is complicated by the additional sources of uncertainty involved in surface micromachining processes. It is not sufficient to use just the experimental error found through repeated measurements in determining the uncertainty of force measurements. Although a measurement may be repeatable, the uncertainty in dimensional and material parameters that influence the spring constant must be included in the error analysis. The purpose of this paper is to define a general-force gauge model that can be used in a design process that considers both estimated force uncertainty and strength-based reliability.

Various methods have been used to measure the forces in surface micromachined devices. Suzuki [1] developed a flexible microgripper that can measure gripping force based upon the driving voltage and elasticity of the microfinger, and claims an accuracy of $1.86 \mathrm{nN}$. Arai et al. [2] have proposed a new method for measuring force using the Laser Raman Spectrophotometer. They expect to achieve a resolution of less than $1 \mu \mathrm{N}$. Xiong et al. [3] describe a balance approach for micro force measurements that is able to measure force and displacement of a cantilever simultaneously.

Other methods of force measurement involve the use of on-chip devices that are fabricated with the devices to be tested. Reid et al. [4] used the amplified deflection of a cantilever beam to measure the force of thermal micro-actuators. A ring-shaped force detector was developed at Sandia National Laboratories [5], where the spring constant was determined experimentally to have an error of about $10 \%$. Yeh and Pister [6] used a serpentine-spring force gauge to measure static 
friction forces. Kruglick and Pister [7] used this same device to measure the applied force in an electric contact study.

Most of the force measurement methods described above used a theoretically exact spring constant to convert a measured displacement to a force. The uncertainty was then found by taking repeated measurements. The methods for measuring the deflection vary, and may include direct optical measurements, interferometry, deflection amplification, or high magnification imaging using scanning electron micrographs. Although the deflection can often be measured accurately, repeated measurements also include variations due to stiction effects and other unknown factors. A source of uncertainty often overlooked is that associated with the spring constant, which depends on many variables such as dimensional and material parameters that are common sources of variation in compliant or flexible mechanisms [8][9]. In addition, the conditions necessary to assume a linear force-deflection relationship, such as small-deflections of linearly elastic, isotropic, homogeneous beams, add to the uncertainty of the force measurements.

Just like any macro gauge, a micro force gauge will have some uncertainty associated with its measurements. Unlike macro gauges, there are no practical means of calibrating micro gauges, so the gauge uncertainty is generally unknown. In many cases there is a temptation to assume exact values for spring dimensions and material properties and base the resolution of the force gauge on the deflection measurement resolution alone. However, proper use of these micro gauges requires some estimation of how dimensional and material variations contribute to the error in force measurements.

This paper first describes a general model for a micromachined force gauge. The accuracy of the gauge is estimated analytically using statistical error propagation methods without the need to assume small deflections. The strength-based reliability [10] (or probability of failure) of the gauge is also determined. Using this probabilistic model in designing a force gauge can then help 


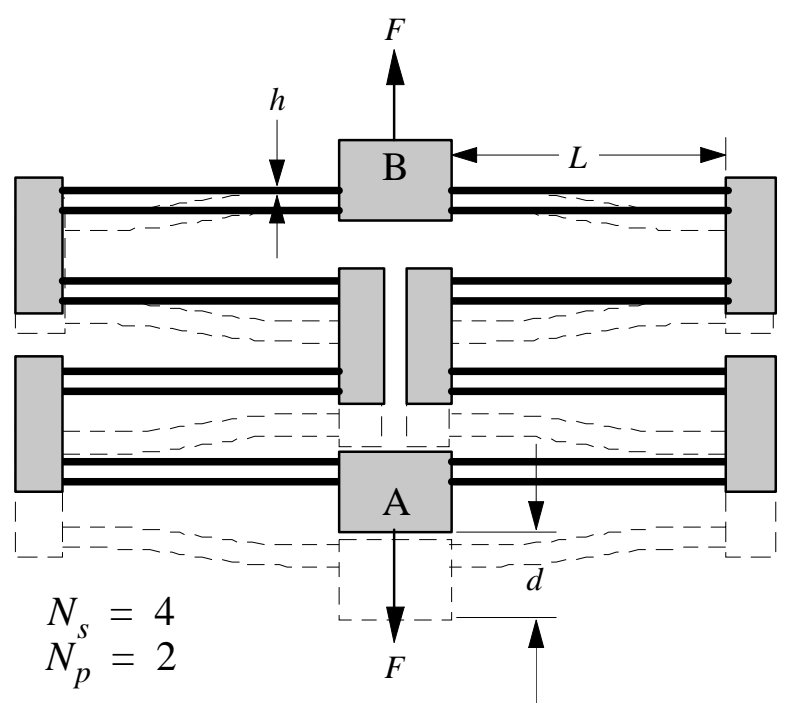

Figure 1: General force gauge model

predict the accuracy and reliability of the gauge for a certain range of force measurements. This method of error analysis is very important at the micro level because of the expense and time involved in fabricating and testing micro devices.

An example is shown of a gauge being used to measure the force required to actuate a bistable mechanism. The force uncertainty is divided into two categories: the experimental error associated with repeated measurements, and the gauge uncertainty due to not knowing the exact values for the dimensions and material properties. These two sources of error are discussed in detail in section 6 .

\section{GENERAL FORCE GAUGE MODEL}

The force gauge described in this paper consists of a symmetric array of beams in parallel and/or series that acts as a spring. The general model is shown in Figure 1 in which a displacement, $d$, is used to determine the applied force, $F . N_{p}$ is the number of beams in parallel, and $N_{s}$ is the number of sets of parallel beams in series on each side of the gauge. The mechanism or system 
to be tested can be connected at point B and the force applied by probe at point A. This model can describe an infinite number of possible configurations, one of which was demonstrated in [6].

\subsection{Small-Deflection Equations}

Figure 2 shows the free-body diagrams used to simplify the force gauge model. Each beam of the force gauge can be modeled as a fixed-guided flexible segment (or constant end angle) with $P=F /\left(2 N_{p}\right)$, and a resultant moment, $M_{o}$. The deflected beam has an inflection point at its center where the curvature is zero. According to the Bernoulli-Euler assumption which states that the moment is directly proportional to the curvature, the moment will be zero at this inflection point. This allows the beam to be simplified to a vertically end-loaded cantilever beam with deflection $\delta=d /\left(2 N_{s}\right)$ and length $l=L / 2$.

The equations for calculating the force come from the simplification of the model to a cantilever beam with applied force, $P$, and deflection, $\delta$, as shown in Figure 2, where $a$ is approximately equal to $L$. For small deflections, the common force-deflection relationship is then 

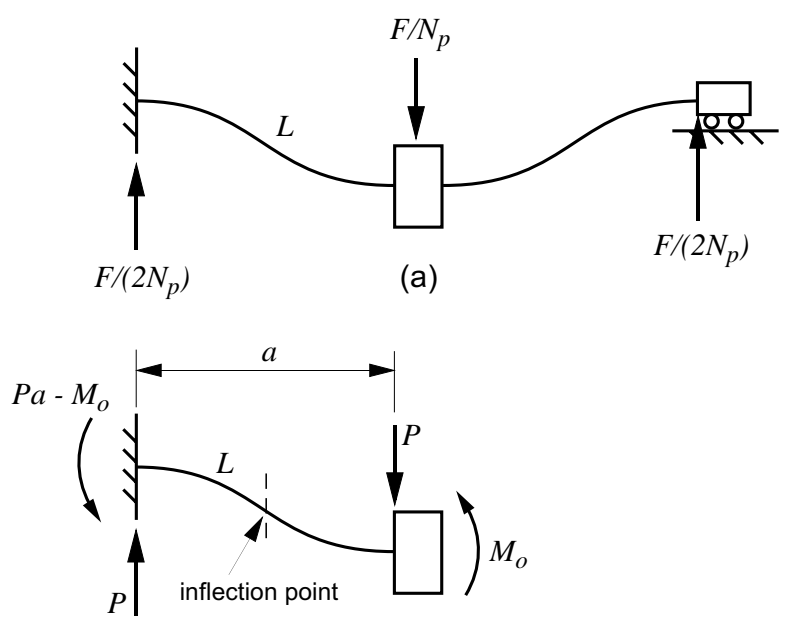

(b)

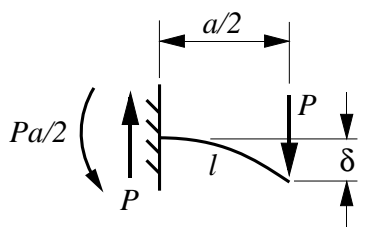

(c)

Figure 2: Free-body diagrams for (a) one 'leg' of the force gauge, (b) a fixed-guidec flexible segment, and (c) a vertically end-loaded cantilever beam.

$\delta=P l^{3} /(3 E I)$. Assuming the cross-section is rectangular, the force and maximum stress for the gauge can be calculated as follows:

$$
\begin{gathered}
F=\frac{N_{p}}{N_{s}} \frac{2 E w h^{3}}{L^{3}} d \\
S_{\text {max }}=\frac{3 E h}{N_{s} L^{2}} d
\end{gathered}
$$

where the moment of inertia, $I$, is $\left(w h^{3}\right) / 12, w$ is out-of-plane thickness of the beam, $h$ is the inplane width of the beam, $E$ is Young's modulus, $d$ is the overall gauge deflection, and $L$ is the length of the beam as shown in Figure 1. 


\subsection{Simplifying the Nonlinear Deflection Equations}

Previous uses of micro force gauges similar to the one described above were limited to small beam deflections. This is because the large deflection of a cantilever beam (Figure 2) is a nonlinear problem that in its exact form is not easily solved or differentiable. In order to evaluate the partial derivatives used in uncertainty analysis, the closed-form nonlinear elliptic integral solution [11] can be simplified to obtain explicit equations that are easily differentiable. This is done by comparing the linear equations (1) and (2) to the elliptic integral solution and evaluating the error as:

$$
\left(F_{\text {linear }}-F_{\text {elliptic }}\right) / F_{\text {elliptic }}
$$

It can be shown that this error is only dependent on the beam deflection, $\delta$, and the beam length, $l$. Error equations can then be found by curve-fitting the error vs. $\delta / l[12]$. For the load case in Figure 2, the error equations for force $\left(\varepsilon_{P}\right)$, and maximum stress $\left(\varepsilon_{S}\right)$, evaluated for $0<\delta / l<0.75$ are:

$$
\begin{aligned}
& \varepsilon_{P}=0.0040\left(\frac{\delta}{l}\right)-1.0760\left(\frac{\delta}{l}\right)^{2}+0.1697\left(\frac{\delta}{l}\right)^{3}-0.3390\left(\frac{\delta}{l}\right)^{4} \\
& \varepsilon_{S}=0.0199\left(\frac{\delta}{l}\right)-0.6969\left(\frac{\delta}{l}\right)^{2}+0.9146\left(\frac{\delta}{l}\right)^{3}-1.1289\left(\frac{\delta}{l}\right)^{4}
\end{aligned}
$$

These curve fits have $R^{2}$ values of 0.9999 and 0.998 , respectively. For $0<\delta / l<0.75$, the percent error for force $(F)$ is less than $0.03 \%$ and less than $0.2 \%$ for the maximum stress $\left(S_{\max }\right)$. These mathematical errors are often negligible when compared to the effects of uncertainty in beam parameters.

Equations (4) and (5) were originally developed to provide a simple means of determining the accuracy of equations (1) and (2) for increasing deflections. For example, if $\delta / l$ is greater than 0.1 , the mathematical force error in the small-deflection equation will be greater than $1 \%$. Since 
these error equations are not dependent upon Young's modulus or the moment of inertia, they can be applied to almost any vertically end-loaded constant cross-section beam that is linearly elastic, homogeneous, and isotropic.

By applying the error equations in an inverse manner to equations (1) and (2), the new equations for determining accurate nominal forces and stresses for deflections up to $\delta / l=0.75$ are:

$$
\begin{gathered}
F=\frac{N_{p}}{2} \frac{E w h^{3} \delta}{l^{3}} \frac{1}{1+\varepsilon_{P}} \\
S_{\text {max }}=\frac{3 E h \delta}{2 l^{2}} \frac{1}{1+\varepsilon_{S}}
\end{gathered}
$$

These equations are still nonlinear with respect to deflection (there is no spring constant), but the nominal values for force and stress are accurate for large deflections, and the partial derivatives used in uncertainty analysis are easy to evaluate. This enables a single gauge to be used for a large range of force measurements.

\section{ERROR ANALYSIS}

The estimation of uncertainty is a stochastic problem that can be approximated using a "partial derivative rule" [10]. This is a common method that is used especially for pre-fabrication estimates or when circumstances prevent an exhaustive statistical study.

\subsection{Variance Approximation}

Given a general function $f\left(\psi_{1}, \psi_{2}, \ldots, \psi_{i}\right)$, where $\psi_{1}, \psi_{2}, \ldots, \psi_{i}$ are independent random variables with standard deviations of $\sigma_{1}, \sigma_{2}, \ldots, \sigma_{i}$, respectively, the estimated variance of $f$ is calculated as: 


$$
\sigma_{f}^{2}=\left(\frac{\partial f}{\partial \psi_{1}}\right)^{2} \sigma_{1}^{2}+\left(\frac{\partial f}{\partial \psi_{2}}\right)^{2} \sigma_{2}^{2}+\ldots+\left(\frac{\partial f}{\partial \psi_{i}}\right)^{2} \sigma_{i}^{2}
$$

where the partial derivatives are calculated using the expected (nominal) values. The variance components on the right-hand side of equation (8) are used to determine the sensitivity of the function with respect to changes in individual random variables. When the function is not highly nonlinear and when the variations are small relative to the nominal values, the estimate of variance is generally assumed to be at least on the right order of magnitude.

All of the variables within equations (6) and (7) have some variation, except for the integers $N_{p}$ and $N_{s}$. The amount of variation in each of the variables will be dependent upon the process used to make the force gauge, with the exception of the beam deflection, $d$. The variation in beam deflection is a result of the uncertainty inherent in the method of measurement, such as optical imaging or interferometry. It should also be emphasized that equation (8) gives only an approximation of the variation that might be expected in actual physical models. Additional factors such as stiction and nonlinear material properties may have significant impact on the actual variation in force and stress measurements.

\subsection{Uncertainty Using the Explicit Equations}

This section shows specifically how the error approximation method can be used in the analysis of the micro force gauge. Using the explicit force gauge equations (6) and (7) for large deflections, the estimated variance for force and stress measurements are calculated as follows:

$$
\begin{gathered}
\sigma_{F}^{2}=\left(\frac{\partial F}{\partial E}\right)^{2} \sigma_{E}^{2}+\left(\frac{\partial F}{\partial w}\right)^{2} \sigma_{w}^{2}+\left(\frac{\partial F}{\partial h}\right)^{2} \sigma_{h}^{2}+\left(\frac{\partial F}{\partial \delta}\right)^{2} \sigma_{\delta}^{2}+\left(\frac{\partial F}{\partial l}\right)^{2} \sigma_{l}^{2} \\
\sigma_{S}^{2}=\left(\frac{\partial S}{\partial E}\right)^{2} \sigma_{E}^{2}+\left(\frac{\partial S}{\partial h}\right)^{2} \sigma_{h}^{2}+\left(\frac{\partial S}{\partial \delta}\right)^{2} \sigma_{\delta}^{2}+\left(\frac{\partial S}{\partial l}\right)^{2} \sigma_{l}^{2}
\end{gathered}
$$

where 


$$
\begin{aligned}
& \frac{\partial F}{\partial E}=\frac{N_{p}}{2} \frac{w h^{3} \delta}{l^{3}} \frac{1}{1+\varepsilon_{P}} \\
& \frac{\partial F}{\partial w}=\frac{N_{p}}{2} \frac{E h^{3} \delta}{l^{3}} \frac{1}{1+\varepsilon_{P}} \\
& \frac{\partial F}{\partial h}=\frac{N_{p}}{2} \frac{3 E w h^{2} \delta}{l^{3}} \frac{1}{1+\varepsilon_{P}} \\
& \frac{\partial F}{\partial \delta}=\frac{N_{p}}{2} \frac{E w h^{3}}{l^{3}}\left[\frac{\left(1+\varepsilon_{P}\right)-\delta\left(\partial \varepsilon_{P} / \partial \delta\right)}{\left(1+\varepsilon_{P}\right)^{2}}\right] \\
& \frac{\partial F}{\partial l}=-\frac{N_{p}}{2} \frac{E w h^{3} \delta}{l^{4}}\left[\frac{3\left(1+\varepsilon_{P}\right)+l\left(\partial \varepsilon_{P} / \partial l\right)}{\left(1+\varepsilon_{P}\right)^{2}}\right] \\
& \frac{\partial S}{\partial E}=\frac{3 h \delta}{2 l^{2}} \frac{1}{1+\varepsilon_{S}} \\
& \frac{\partial S}{\partial h}=\frac{3 E \delta}{2 l^{2}} \frac{1}{1+\varepsilon_{S}} \\
& \frac{\partial S}{\partial \delta}=\frac{3 E h}{2 l^{2}}\left[\frac{\left(1+\varepsilon_{S}\right)-\delta\left(\partial \varepsilon_{S} / \partial \delta\right)}{\left(1+\varepsilon_{S}\right)^{2}}\right] \\
& \frac{\partial S}{\partial l}=-\frac{3 E h \delta}{2 l^{3}}\left[\frac{2\left(1+\varepsilon_{S}\right)+l\left(\partial \varepsilon_{S} / \partial l\right)}{\left(1+\varepsilon_{S}\right)^{2}}\right]
\end{aligned}
$$

and for $i=P$ and $i=S$,

$$
\begin{aligned}
& \frac{\partial \varepsilon_{i}}{\partial \delta}=\frac{C_{i 1}}{l}+\frac{2 C_{i 2} \delta}{l^{2}}+\frac{3 C_{i 3} \delta^{2}}{l^{3}}+\frac{4 C_{i 4} \delta^{3}}{l^{4}} \\
& \frac{\partial \varepsilon_{i}}{\partial l}=-\frac{C_{i 1} \delta}{l^{2}}-\frac{2 C_{i 2} \delta^{2}}{l^{3}}-\frac{3 C_{i 3} \delta^{3}}{l^{4}}-\frac{4 C_{i 4} \delta^{4}}{l^{5}}
\end{aligned}
$$


where $\mathrm{C}_{i 1}, \mathrm{C}_{i 2}, \mathrm{C}_{i 3}$, and $\mathrm{C}_{i 4}$ are the coefficients of equations (4) and (5). Although equation (10) and the associated partial derivatives (16) through (21) can be used to estimate the uncertainty in stress measurements, it is often more useful to use these results to estimate the strength-based reliability, as discussed in the next section.

\section{PROBABILISTIC STRENGTH-BASED RELIABILITY}

Both the fracture strength and the predicted maximum stress are random variables. Assuming that they are normally distributed about their expected values, the probability of failure due to exceeding the fracture strength of the material can be estimated analytically. Using a $\mathrm{z}$ value of

$$
z=\frac{-\left(S_{y}-S_{\max }\right)}{\sqrt{\sigma_{S_{y}}^{2}+\sigma_{S}^{2}}}
$$

the probability that a beam will fracture at a given displacement can be calculated by integrating the normal probability density function from $-\infty$ to $z$ :

$$
P[\text { fracture }]=\Phi(z)=\int_{-\infty}^{z} \frac{1}{\sqrt{2 \pi}} e^{-t^{2} / 2} d t
$$

This can be very useful during the design of the gauge to determine its approximate reliability, especially if the fracture strength is determined experimentally and the experimental mean and standard deviation are available. (The equation for stress variance, $\sigma_{S}^{2}$, includes the uncertainty in the measured beam deflection, $\sigma_{\delta}$. However, when calculating the probability of failure for a specific displacement, $\sigma_{\delta}$ is zero.)

It should be emphasized that the probability of failure is only an estimate. Although effects of stress concentration factors and grain sizes are often included in the fracture strength 


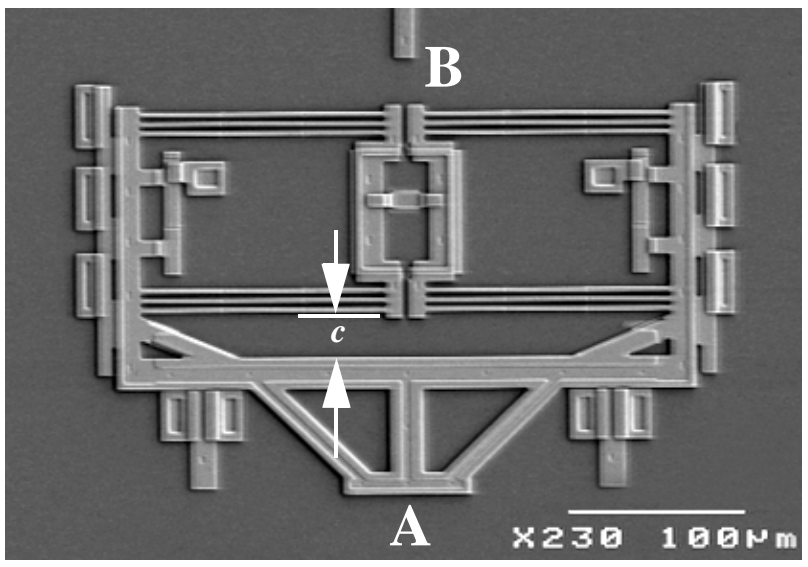

Figure 3: Micrograph of a force gauge.

variation, there may be other factors not considered such as nonlinear stress-strain relationships and stiction loads.

\section{EXAMPLE}

The force gauge shown in Figure 3 has been designed for testing various types of actuators with forces in the range of 100 to $1000 \mu \mathrm{N}$. By pushing at point A with a probe, the end of the actuator contacting at B will cause the parallel beams to deflect. The force is determined by measuring the gauge deflection, $d=25-c$ (where the undeflected position is $c=25$ microns), and using equations (6) and (7) to find the nominal force and maximum stress. The parameters for the mechanism are: $L=150 \mu \mathrm{m}, w=3.5 \mu \mathrm{m}, h=3.0 \mu \mathrm{m}, E=162 G P a, S_{y}=1.56 G P a, N_{p}=6$, and $N_{s}$ $=1$.

\subsection{Values for $\sigma_{i}$}

The material property and dimensional variations will vary with the process used to fabricate the force gauges. In this example, the force gauge was fabricated using MUMPs ${ }^{\mathrm{TM}}$. For this process, Sharpe and Jackson [13] have measured a value of $162 \pm 14 \mathrm{GPa}$ for Young's modulus and $1.56 \pm 0.25 \mathrm{GPa}$ for tensile strength. Assuming typical engineering practice of plus/minus 
values at the $95 \%$ confidence limit, the standard deviations for Young's modulus and tensile strength are approximately $\sigma_{E}=7.143 \mathrm{GPa}$ and $\sigma_{S y}=0.128 \mathrm{GPa}$.

Both the polysilicon (Poly1 and Poly2) and the sacrificial oxide layers are grown using low pressure chemical vapor deposition. This is a timed process designed to produce a nominal thickness for each layer. The tolerances for the individual layer thicknesses can be found in the MUMPs ${ }^{\mathrm{TM}}$ Design Handbook [14]. The standard deviation is taken to be approximately one third of the tolerance. For $w=2.0 \mu \mathrm{m}$ (Poly1), $\sigma_{w}=0.050 \mu \mathrm{m}$. For $w=1.5 \mu \mathrm{m}$ (Poly2), $\sigma_{w}=0.033 \mu \mathrm{m}$. For $w=3.5 \mu \mathrm{m}\left(\right.$ Poly1+Poly2), $\sigma_{w}=0.060 \mu \mathrm{m}$.

The geometry in the various polysilicon and oxide layers is created using a Reactive Ion Etch (RIE). Although the masks can be made very precise, the variables in the etch process lead to variations in the smoothness and angle of the side-walls [8]. A side-wall angle between 89 and 90 degrees combined with the surface roughness results in approximately $\sigma_{h}=0.05 \mu \mathrm{m}$. Although the variation in the beam lengths is very small, a value of $0.1 \mu \mathrm{m}$ will be used for $\sigma_{L}$. This results in $\sigma_{l}=\sigma_{L} / 2=0.05 \mu \mathrm{m}$.

In addition to uncertain material and dimensional parameters, a known source of error is the uncertainty in the displacement measurement. This will vary greatly depending upon the equipment being used. Using an optical microscope, a conservative value for the standard deviation in the displacement measurement is $\sigma_{d}=0.5 \mu \mathrm{m}$. This results in $\sigma_{\delta}=\sigma_{d} /\left(2 N_{s}\right)=0.25 \mu \mathrm{m}$. 
TABLE 1: Estimated force and stress results at various measured deflections.

\begin{tabular}{|r|r|r|r|r|}
\hline & \multicolumn{4}{|c|}{ Deflection $(d)$} \\
& $2 \mu \mathrm{m}$ & $10 \mu \mathrm{m}$ & $15 \mu \mathrm{m}$ & $20 \mu \mathrm{m}$ \\
\hline$F$ & 109 & 547 & 825 & 1109 \\
\hline$\sigma_{F}$ & 28 & 47 & 63 & 82 \\
\hline$\%$ Error & $26 \%$ & $9 \%$ & $8 \%$ & $7 \%$ \\
\hline \multicolumn{4}{|c|}{} \\
\hline$S_{\max }$ & 130 & 649 & 976 & 1306 \\
\hline$\sigma_{S}$ & 33 & 45 & 57 & 70 \\
\hline$\%$ Error & 0 & 0 & 0 & 0 \\
\hline P[fracture] & $<1 \%$ & $<1 \%$ & $<1 \%$ & $4 \%$ \\
\hline
\end{tabular}

\subsection{Results}

Table 1 shows the results of the error analysis for force and stress at different measured deflections. The standard deviations for force and stress, $\sigma_{F}$ and $\sigma_{S}$, were calculated using the method described in section 3.2. The uncertainties are then represented as the nominal value \pm the standard deviation, or $F \pm \sigma_{F}$ and $S_{\max } \pm \sigma_{S}$. The percent error is the ratio of the standard deviation over the nominal value. The following observations can be made from Table 1:

- This particular gauge design is not well suited to making accurate measurements at small deflections.

- Even though the maximum stress is below the fracture strength, there is still about a $4 \%$ chance of fracture at a gauge deflection of $20 \mu \mathrm{m}$.

A better understanding of how variation in the individual parameters affects the force is gained from a sensitivity analysis. This is done by comparing the terms on the right hand side of equations (9) and (10). The percent contribution is found by dividing each term by the total vari- 
Force Error vs. Gauge Deflection

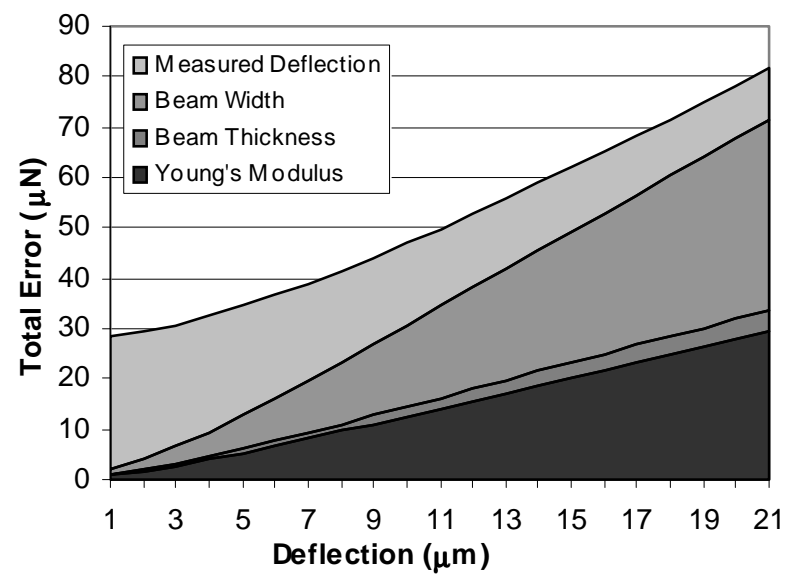

Figure 4: Relative contributions of the variation in $\delta, h$, $w$, and $E$ to the total force error at different deflections.

ance. Table 2 shows the variance components evaluated at a gauge deflection of $20 \mu \mathrm{m}$. It is evident that the variance component associated with uncertainty in length is insignificant compared to that of the other random variables.

It is important to determine if the force error is constant or whether it changes with gauge deflection. The total force error at different gauge deflections is shown in Figure 4 along with the relative contributions of the variation in measured beam deflection $\left(\sigma_{\delta}\right)$, in-plane width $\left(\sigma_{h}\right)$, beam thickness $\left(\sigma_{w}\right)$, and Young's modulus $\left(\sigma_{E}\right)$. The error due to variation in length was nearly zero, so it is excluded. This graph shows that the error due to $\sigma_{h}, \sigma_{w}$, and $\sigma_{E}$ increases linearly with increasing deflection, while the error due to measured beam deflection increases nonlinearly.

TABLE 2: Estimated variance components for force and stress at a measured deflection of $20 \mu \mathrm{m}$.

\begin{tabular}{|c|r|r|r|r|}
\hline & \multicolumn{2}{|c|}{ Force } & \multicolumn{2}{c|}{ Stress } \\
& Value & Contribution & Value & Contribution \\
\hline $\operatorname{Var}_{E}$ & 2391 & $36 \%$ & 3318 & $68 \%$ \\
\hline $\operatorname{Var}_{w}$ & 361 & $5 \%$ & $\mathrm{n} / \mathrm{a}$ & $\mathrm{n} / \mathrm{a}$ \\
\hline $\mathrm{Var}_{h}$ & 3074 & $46 \%$ & 474 & $10 \%$ \\
\hline $\operatorname{Var}_{\delta}$ & 827 & $12 \%$ & 1105 & $23 \%$ \\
\hline $\operatorname{Var}_{I}$ & 5 & $<1 \%$ & 3 & $<1 \%$ \\
\hline $\mathrm{Var}_{\text {Total }}$ & 6659 & & 4900 & \\
\hline
\end{tabular}




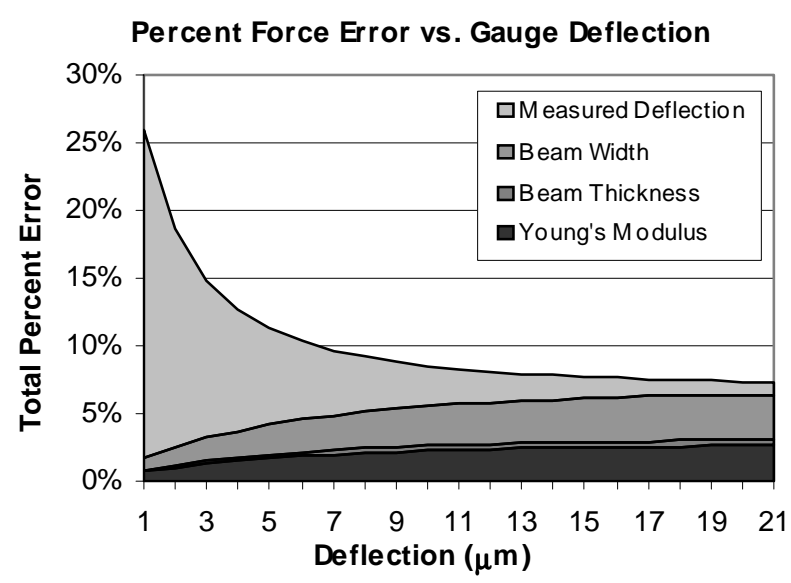

Figure 5: Contribution of the relative variation in $\delta, h, w$, and $E$ to the overall force uncertainty at different deflections.

Another way of comparing the variance components is by looking at the percent error. It is a simple matter to show that if $\sigma_{\delta}$ is taken to be zero, the percent error due to $\sigma_{h}, \sigma_{w}$, and $\sigma_{E}$ is a constant value of about $7 \%$ in this example. However, including $\sigma_{\delta}$ in the analysis affects the error significantly for small deflections. Figure 5 shows the percent force error vs. gauge deflection and the relative contributions of the different parameter variations. From this graph, the following observations can be made:

- For small deflections, the force error is dominated by the uncertainty in the deflection measurement.

- For large deflections, the force error is dominated by the uncertainty in the in-plane width $(h)$ and Young's modulus.

These results would suggest that the first step in reducing the (percent) gauge uncertainty is to design the gauge for large deflections in the range of force that is required. Any further reduction would require knowing Young's modulus with more certainty, reducing the beam width variation $\left(\sigma_{h}\right)$, or performing design synthesis on the mechanism coupled with optimization to give the best combination of $h, w, L, N_{s}$, and $N_{p}$. Choosing a design to give a minimum uncer- 
tainty is therefore not trivial, but as a general rule, decreasing the ratio of $\sigma_{i}$ to the nominal parameter value will also decrease the force uncertainty. This means that if nothing can be done to alter the uncertainty in Young's modulus, the force uncertainty can still be decreased by increasing $h$.

An essential part of the force gauge design is estimating the reliability at its maximum specified deflection, using the method described in section 4. Equation (10) shows that the probability of failure is dependent upon $S_{y}, S_{m a x}$, and their variances, $\sigma_{S y}{ }^{2}$ and $\sigma_{S}{ }^{2}$. From a design standpoint, $S_{y}$ and $\sigma_{S y}{ }^{2}$ cannot be changed, as these are dependent on the manufacturing process and material properties. Therefore, in order to modify the mechanism's probability of failure, a designer may only directly modify $S_{\max }$ and $\sigma_{S}^{2}$.

To lower the probability of failure, it is desirable to design the gauge to yield a large negative z-value (a z-value of zero would correspond to a 50\% chance of failure). A large z-value can be obtained by decreasing both $S_{\max }$ and $\sigma_{S}{ }^{2}$. Equation (7) shows that $S_{\max }$ can be decreased by either decreasing $h$, decreasing $\delta$, and/or increasing $l$. It is important to note that a change in $l$ will be more significant than a proportional change in $\delta$ or $h$. This means that $h$ can be increased to reduce force uncertainty if $l$ is also increased to maintain reliability requirements.

Reliability can also be improved by decreasing the total stress variance. Table 2 shows the predicted variance components for the maximum stress (evaluated at a maximum measured gauge deflection of $20 \mu \mathrm{m})$. It can be seen that Young's modulus contributes about $68 \%$ of the total variance. However, it must be emphasized that it is not the percent error in stress measurement that is used to predict the reliability, but the total variance, $\sigma_{S}{ }^{2}$, evaluated at an exact specified displacement $\left(\sigma_{\delta}=0\right)$. Fortunately, equations (16) through (19) reveal that the same changes in design parameters that decrease $S_{\text {max }}$ will also decrease $\sigma_{S}{ }^{2}$. Therefore, analyzing the individual variance components for stress is not important. 
The main complication in designing this gauge is balancing the force uncertainty and reliability. For example, decreasing the in-plane width $(h)$ or deflection $(\delta)$ will increase the reliability, but this also increases force uncertainty. On the other hand, increasing the beam length, $l$, improves both the reliability and the uncertainty. Since large deflections are desirable to decrease percent force error, the best alternative is to either increase the beam length, or increase $N_{S}$ so that the beam deflection, $\delta$, can be decreased without reducing the overall gauge deflection, $d$. However, additional consideration must be given to the possibility of other unknown factors that may influence the behavior of the gauge, such as stiction. For example, long slender beams are often more susceptible to stiction problems than short stiff beams.

\section{EXPERIMENTAL ERROR VS. GAUGE UNCERTAINTY}

Up to this point, the design and error analysis of the micro gauge has considered both the error due to inaccuracy in deflection measurements and the uncertainty in material and dimensional parameters. However, the predicted gauge uncertainty should not be confused with experimental error. Once a gauge is fabricated it will have set dimensions and material properties. Because it is not practical to determine their exact values, this uncertainty contributes to an unknown mean shift in the calculated forces (i.e. gauge uncertainty). In contrast, the deflection measurement uncertainty will be part of the experimental error. There may also be other factors contributing to the experimental error that cannot be easily predicted.

As an example, we will consider the results of an experiment where a gauge has been used to determine the force required to actuate a bistable mechanism. From repeated measurements, the average force was $370 \mu \mathrm{N}$ with a standard deviation of $27 \mu \mathrm{N}$. The predicted uncertainty of the gauge before fabrication, using the techniques described above, was $31 \mu \mathrm{N}$. However, part of this estimation of error was due to uncertainty in the measured deflection. As described above, the 
gauge uncertainty when considering only the mean shift, is a constant $7 \%$, or about $26 \mu \mathrm{N}$. The experimental error of $27 \mu \mathrm{N}$ and the gauge uncertainty of $26 \mu \mathrm{N}$ combine by root-sum-square to give an overall uncertainty of about $37 \mu \mathrm{N}$.

This paper focuses on predicting the gauge uncertainty, but it is also often helpful to compare the experimental repeatability to the other quantifiable sources of error. For example, the predicted experimental error due to uncertainty in measured deflection $(d)$ alone, was about $18 \mu \mathrm{N}$. Formal statistical inference methods can then be used to determine if the experimental error of 27 $\mu \mathrm{N}$ indicates the presence of significant unknown factors.

This example shows that the gauge uncertainty can be significant when compared to experimental repeatability, and should be included in the overall force uncertainty. Also, the gauge uncertainty (due to Young's modulus and dimensional parameters) is a constant percentage over the range of deflection, rather than a set standard deviation. This means that for small deflections, the experimental repeatability may dominate the force error, but with increasing deflection, the gauge uncertainty may become the dominating source of error. These two sources are independent, with one being experimentally determined, and the other being estimated using the methods described in this paper. The significance of other unknown factors affecting the experimental error can be determined through comparison to the uncertainty due to measured deflection.

\section{GENERAL DESIGN GUIDELINES}

The general force gauge model described above can be used to measure a very large range of forces. Since the governing equations are under-constrained, the design space is infinite. To design a gauge to measure a particular range of forces, the designer may choose additional constraints such as the overall size of the gauge. It is not a trivial matter to obtain an optimal design, and even more difficult to prove if an optimum really exists, due to the many unknown factors 
involved in the experimental error. However, it is not difficult to develop a suitable design through iteration, if the following steps are followed:

1. Determine the process tolerances and uncertainties to obtain values for $\sigma_{i}$.

2. Choose a reliability level such as a probability of failure less than $5 \%$.

3. Choose initial values of the parameters to meet the reliability requirement and ensure that $0<\delta / l<0.75$.

4. Choose values for $w, N_{s}$, and $N_{p}$ to match the desired force measurement range.

5. Adjust the parameters to balance between force uncertainty, reliability, overall mechanism size, and any other factors that may be important.

The fifth step is the most difficult, but a sensitivity or variance analysis can be extremely helpful in determining which parameters to adjust to improve accuracy and reliability. Consideration must be given to the possibilities of other factors such as stiction effects, nonlinear material properties, stress concentrations, etc. Also, when the gauge is designed to be contracted, or "pushed", care must be taken to ensure that the maximum deflection of the beams does not cause interference. When the force gauge is designed to be "pulled", the spacing between the beams is not as important.

As an example of reducing force uncertainty, consider a gauge that is designed to reduce the error for the force range of 100 to $200 \mu \mathrm{N}$. For the gauge described in the example of section 5, it was shown that the percent error is high for small forces due to the inaccuracy in measuring the deflection. For example, the predicted errors in measuring forces of 100 and $200 \mu \mathrm{N}$ are about $26 \%$ and $16 \%$, respectively. These errors can be reduced to $11 \%$ and $8 \%$ by simply increasing $N_{S}$ to 3 . The error can be reduced to about $6 \%$ over the full range of 100 to $200 \mu \mathrm{N}$ by making additional changes to the beam dimensions: $L=200.0 \mu \mathrm{m}, w=2.0 \mu \mathrm{m}$, and $h=4.0 \mu \mathrm{m}$. This demonstrates that the estimated gauge uncertainty can be reduced by making simple changes to the 
gauge design. Although these theoretical predictions do not consider all possible sources of error, the methods discussed can be used to reduce the effects of known variations.

\section{CONCLUSION}

The general force gauge model described in this paper can be used to measure a wide range of forces. If the material properties and dimensional parameters are treated as random variables, the gauge uncertainty and reliability can be estimated before fabrication. This can aid in the design of gauges specific to certain force ranges, with increased accuracy and reliability.

The error analysis methods in this paper are not limited to small beam deflections. Using error-correction equations, explicit equations were found that accurately predict the nominal force and stress in large-deflection cantilever beams. This allows a single gauge to be used in measuring a wider range of force.

The reliability of the force gauge can be estimated using a probabilistic strength-based approach. The same methods for determining the error in force measurements can be used to estimate the maximum stress for a given deflection. If the mean and standard deviation of the fracture strength are known, the probability of failure can then be estimated.

There are many possible designs for measuring the same range of forces. Finding the optimal combination of $L, h, w, E, N_{S}$, and $N_{p}$ to reduce the gauge uncertainty is not a trivial matter, but it is often only necessary to develop a suitable design that meets some set of specifications such as a minimum precision and maximum size. Some of the general guidelines that can aid in design are listed below:

- Increasing $N_{p}$ increases the force without altering the maximum stress or the error.

- Where it is not possible to measure deflections accurately, $N_{s}$ can be increased to reduce the effects of this error. 
- For extremely small forces, minimize $N_{p}$ and maximize $N_{s}$ and $l$ within the design constraints.

- Increasing the reliability while maintaining the force range can be achieved by increas$\operatorname{ing}$ both $N_{s}$ and $N_{p}$ (this reduces the beam deflection, $\delta$, required for measuring a given force).

- The force uncertainty can be decreased while maintaining reliability if both $h$ and $l$ are increased.

- Ultimately, the force accuracy will be limited by the uncertainty in Young's modulus.

The error in force measurements due to gauge uncertainty (variations in material properties and dimensional parameters) was found to be significant when compared with measurement repeatability, especially for large deflections. A known source of experimental error is the inaccuracy in the measurement of gauge deflection, but this is often insufficient to account for all of the error. Further studies ought be performed to determine how to account for other factors that may affect experimental force measurements.

Without a means of calibrating force gauges, the methods described in this paper provide at least an approximation for the gauge uncertainty and reliability. Until more work is done to better quantify the actual material properties and dimensional variations, it is advisable to be conservative when reporting the accuracy and precision of micro force measurements.

\section{ACKNOWLEDGEMENTS}

The authors gratefully acknowledge the support of the National Science Foundation through NSF grants DMI-9624574, CMS-9978737, and an NSF graduate research fellowship. 


\section{REFERENCES}

[1] Suzuki, Y., 1996, "Flexible Microgripper and its Application to Micromeasurement of Mechanical and Thermal Properties," Proceedings of the IEEE Micro Electro Mechanical Systems (MEMS), Feb 11-15, pp. 406-411.

[2] Arai, F., Nonoda, Y., and Oota, T., 1996, "New Force Measurement and Micro Grasping Method Using Laser Raman Spectrophotometer," Proceedings of the 1996 IEEE International Conference on Robotics and Automation, pp. 2220-2225.

[3] Xiong, X., Zou, Q., Lu, D., and Wang, W., 1998, "Balance-Approach for Load-Displacement Measurement of Microstructures," Mechatronics, Vol. 8, No. 5, pp. 549-559.

[4] Reid, J.R., Bright, V.M., and Comtois, J.H., 1996, "Force measurements of polysilicon thermal micro-actuators," SPIE, Vol. 2882, pp 296-306.

[5] Tanner, D.M., et al., 2000, "MEMS Reliability: Infrastructure, Test Structures, Experiments, and Failure Modes," Sandia National Laboratories, Sandia Report SAND2000-0091.

[6] Yeh, R., and Pister, K.S.J., 1995, "Measurement of Static Friction in Mechanical Couplings of Articulated Microrobots," SPIE Micromachined Devices and Components, Vol. 2642, pp. $40-50$.

[7] Kruglick, E.J.J., and Pister, K.S.J., September, 1999, "Lateral MEMS Microcontact Considerations," Journal of Microelectromechanical Systems, Vol. 8, No. 3, pp. 264-271.

[8] Mirfendereski, D., Lin, L., Kiureghian, A.D., and Pisano, A.P., 1993, "Probabilistic Response of Micro-Fabricated Polysilicon Beam Structures: Comparison of Analysis and Experiments," ASME, Dynamic Systems and Control Division, Vol. 46, pp. 77-80.

[9] Howell, L. L., Rao, S. S., and Midha, A., 1994, "Reliability-Based Optimal Design of a Bistable Compliant Mechanism," ASME Journal of Mechanical Design, December, Vol. 116, No. 1, pp. 1115-1121. 
[10] Rao, S.S., 1992, Reliability-Based Design, McGraw-Hill, Inc., New York.

[11] Bisshopp, K.E., and Drucker, D.C., 1945, "Large Deflection of Cantilever Beams," Quarterly of Applied Mathematics, Vol. 3, No. 3, pp. 272-275.

[12] Wittwer, J.W., 2001, "Predicting the Effects of Dimensional and Material Property Variations in Micro Compliant Mechanisms," M.S. Thesis, Brigham Young University, Provo, Utah.

[13] Sharpe, W.N., Turner, K.T., and Edwards, R.L., 1999, "Tensile Testing of Polysilicon," Experimental Mechanics, Vol. 39, No. 3, pp. 162-170.

[14] Koester, D.A., Mahadevan, R., Hardy, B., and Markus, K.W., 2001, MUMPs ${ }^{\mathrm{TM}}$ Design Handbook, Revision 6.0, Cronos Integrated Microsystems. 